International Journal of Instrumentation and Control Systems (IJICS) Vol.7, No.3, July 2017

\title{
DESIGN OF PID CONTROLLERS INTEGRATOR SYSTEM WITH TIME DELAY AND DOUBLE INTEGRATING PROCESSES
}

\author{
B.S.Patil ${ }^{1}$, L.M.Waghmare ${ }^{2}$, M.D.Uplane ${ }^{3}$ \\ ${ }^{1}$ Ph.D.Student, Instrumentation Department, \\ AISSMS'S Polytechnic, Pune, Maharashtra, India \\ ${ }^{2}$ Guide, Instrumentation Department, Shri Guru Gobind Singhji Institute of Engineering \\ \& Technology, Vishnupuri, Nanded, Maharashtra, India. \\ ${ }^{3}$ Co-guide, Instrumentation Science Department, \\ Savitribai Phule Pune University, Pune, Maharashtra, India
}

\begin{abstract}
In this paper first we investigate optimal PID control of a double integrating plus delay process and compare with the SIMC rules. What makes the double integrating process special is that derivative action is actually necessary for stabilization. In control, there is generally a trade-off between performance and robustness, so there does not exist a single optimal controller. However, for a given robustness level (here defined in terms of the Ms-value) we can find the optimal controller which minimizes the performance $J$ (here defined as the integrated absolute error (IAE)-value for disturbances). Interestingly, the SIMC PID controller is almost identical to the optimal pid controller. This can be seen by comparing the paretooptimal curve for $J$ as a function of Ms, with the curve found by varying the SIMC tuning parameter Tc.

Second, design of Proportional Integral and Derivative (PID) controllers based on internal model control (IMC) principles, direct synthesis method (DS), stability analysis (SA) method for pure integrating process with time delay is proposed. The performances of the proposed controllers are compared with the controllers designed by recently reported methods. The robustness of the proposed controllers for the uncertainty in model parameters is evaluated considering one parameter at a time using Kharitonov's theorem. The proposed controllers are applied to various transfer function models and to non linear model of isothermal continuous copolymerization of styrene-acrylonitrile in CSTR. An experimental set up of tank with the outlet connected to a pump is considered for implementation of the PID controllers designed by the three proposed methods to show the effectiveness of the methods.
\end{abstract}

\section{KEYWORDS}

PID control, optimization, double integrating plus time delay, SIMC rules, integrating systems, internal model controller, stability analysis, direct synthesis method, robustness, Kharitonov's theorem, level control in cylindrical tank.

\section{INTRODUCTION}

A simple IMC-PID controller design technique is proposed on the basis of the IMC principle for two representative integrating processes with time delay. Further, it is extended to integrating

DOI : 10.5121/ijics.2017.7301 
International Journal of Instrumentation and Control Systems (IJICS) Vol.7, No.3, July 2017

processes with negative and positive zero as well. The proposed PID controller design method is mainly focused on the disturbance rejection, which causes the overshoot in the set-point response, and a two-degree-of-freedom (2DOF) control structure is used to eliminate this overshoot. The simulation results show the superiority of the proposed tuning rule over other existing methods, when the controller is tuned to have the same robustness level by evaluating the peak of the maximum sensitivity (Ms). The closed loop time constant $(\lambda)$ has only one user-defined tuning parameter in the proposed method. A guideline is suggested for the selection of $\lambda$ for different robustness levels by evaluating the value of Ms Over a wide range of $\theta / \tau$ ratios

Integrating systems with time delay are found in the modeling of liquid level systems, liquid storage tanks, boilers, batch chemical reactors and the bottom level control of a distillation column [1]. Chien and Freuhauf [1] have suggested that many chemical processes can be modeled for the purpose of designing controllers by a transfer function Fuentes and Luyben [2] have reported that the composition control loop of a high purity distillation column has a large time constant and hence, the response resembles that of a pure integrator plus dead time model. An isothermal continuous copolymerization reactor can be modeled as an integrating system with dead time [3]. The model contains only two parameters ( $k$ and $L p$ ) and the model is very simple for identification. The model is able to adequately represent the dynamics of many systems over the frequency range of interest for the PID controller design. In industries $95 \%$ of the controllers are of PID type $[4,5]$.

Many units used in the chemical process industry, such as heat-ing boilers, batch chemical reactors, liquid storage tanks or liquid level systems, are integrating processes in which the dynamic response is very slow with a large dominant time constant. Due to transportation delays in the recycle loops and composition analysis loops etc., a time delay exists in the majority of processes used in the process industries. In process control, the majority of the control loops are of the proportional-integral-derivative (PID) type at the regulatory level. The main reason for this is their relatively simple structure, which can be readily understood and allows them to be easily implemented in practice. Finding design methods that lead to the optimal operation of the PID controllers is therefore of significant interest. Integrating processes or first order systems with an integrator (with/without zero) are frequently encountered in the process industries. For first order systems with an integrator and with/without zero, if the zero is positive, the system exhibits an inverse response; if the zero is negative, then the system shows large overshoot in the response.

\section{LITERATURE SURVEY}

\subsection{PID controller design for integrating processes with time delay. (2008)}

Authors: Shamsuzzoha, Md. and Lee, M.

Description: In this paper, design of Proportional Integral and Derivative (PID) controllers based on internal model control (IMC) principles, direct synthesis method (DS), stability analysis (SA) method for pure integrating process with time delay is proposed. The performances of the proposed controllers are compared with the controllers designed by recently reported methods. The robustness of the proposed controllers for the uncertainty in model parameters is evaluated considering one parameter at a time using Kharitonov's theorem. The proposed controllers are applied to various transfer function models and to non linear model of isothermal continuous copolymerization of styrene-acrylonitrile in CSTR. An experimental set up of tank with the outlet 
International Journal of Instrumentation and Control Systems (IJICS) Vol.7, No.3, July 2017

connected to a pump is considered for implementation of the PID controllers designed by the three proposed methods to show the effectiveness of the methods.

\subsection{Optimal PI-control and verification of the SIMC tuning rule. (2012)}

Authors: Grimholt, C. and Skogestad, S.

Description: Optimal PI-settings are derived for first-order with delay processes for specified levels of robustness (Ms-value) and compared with the simple SIMC-rule. Optimality (performance) is defined in terms of the integrated absolute error (IAE) of the output for combined step changes in set-points and input disturbances. With SIMC, the robustness level is adjusted by changing the tuning parameter $\tau \mathrm{c}$, and the SIMC-rule was found to give surprisingly good setting with almost Pareto-optimal performance. The exception is a pure time delay processes where the SIMC-rule gives a pure integral controller with somewhat sluggish response. A simple modification to improve on this is to increase the time constant in the rule by one third of the time delay.

\subsection{Identification and Tuning of Integrating Processes with Dead time and Inverse Response.}

Author- William L. Luyben.

Description: The classical example of an integrating method with inverse reply is level control of a boiler steam drum. The "boiler swell" difficulty can lead to a transfer function between the drum level and boiler feed water flow rate that contains a clean integrator and a positive zero, in addition to some dead time and lags. A procedure for identify the transfer-function parameters for this type of structure from step response data. A proportional integral controller tuning process is also presented. Because the process contains an integrator and the proportional-integral controller also contains an integrator, manager tuning is somewhat complex.

\subsection{Design of PID Controllers for Improved Performance of Higher Order Systems.}

Author- L. M. Waghmare G. M. Malwatkar.

Description: Frequency reply based design method of PID controller is proposed for higher order (HO)/-plus delay point in time (HOPDT) systems. The HO/-PDT models are converted into real and unreal part at a incidence where a criteria similar to amplitude and phase margin is utilized to get the constraints on the parameter of the controllers. To discard sound and load disturbances, the constraint given by sensitivity and complementary sensitivity function are also considered. The proposed method has larger features for systems with high order, time delays and monotonic or oscillatory responses.

\subsection{On Tuning PI Controllers for Integrating Plus Time Delay Systems. (2010)}

Authors: Di Ruscio, D.

Description: This paper is concerned about PI controller tuning based on integrator plus time delay models. Integrator plus time delay processes and close to integrator plus time delay systems are common and important processes in industry. Examples of integrating plus time delay 
International Journal of Instrumentation and Control Systems (IJICS) Vol.7, No.3, July 2017

processes are level systems, pulp and paper plants, oil-water-gas separators in oil industry, and all time constant lag dominant processes which may be approximated with an integrator plus time delay process.

\section{PROPOSED SYSTEM}

In this system, design of Proportional Integral and Derivative (PID) controllers based on internal model control (IMC) principles, direct synthesis method (DS), stability analysis (SA) method for pure integrating process with time delay is proposed. The performances of the proposed controllers are compared with the controllers designed by recently reported methods. The robustness of the proposed controllers for the uncertainty in model parameters is evaluated considering one parameter at a time using Kharitonov's theorem. The proposed controllers are applied to various transfer function models and to non linear model of isothermal continuous copolymerization of styrene-acrylonitrile in CSTR. An experimental set up of tank with the outlet connected to a pump is considered for implementation of the PID controllers designed by the three proposed methods to show the effectiveness of the methods also in this system we are using ultrasonic sensor to sense the water level that sensor is connected to the ardino uno which take signal process it give signal to relay according to signal water motor gets on.

\section{Motivation:}

$>$ The ubiquitous PID controller has continued to be the most usually used process control technique for many decades. Although advanced control techniques such as model predictive control can supply significant improvements, a PID controller that is properly designed and tune has proved to be suitable for the vast majority of industrial control loops.

$>$ The enormous literature on PID controllers includes a wide selection of design and tuning methods based on different performance criteria Incorporating systems with time suspension are create in the model of liquid level system, liquid storage tank, boilers, batch chemical reactors and the bottom level manage of a distillation column.

\section{Methods in Proposed System}

The present work is intended to design PID controllers for pure integrating systems with time delay using three methods (i) IMC method (ii) direct synthesis method and (iii) stability analysis method.

\section{IMC Method}

A well-known control system design strategy, internal model control (IMC) was developed by Morari and co-workers2 0 and is closely related to the direct synthesis approach. Like the DS method, the IMC method is based on an assumed process model and relates the controller settings to the model parameters in a straightforward manner. The IMC approach has the advantages that it makes the consideration of model uncertainty and the making of tradeoffs between control system performance and robustness easier.

The process transfer function is given by 
International Journal of Instrumentation and Control Systems (IJICS) Vol.7, No.3, July 2017

$$
G_{p}=\frac{k_{p} e^{-L s}}{s}
$$

Using Pade's approximation for time delay, Eq (1) is rewritten as

$$
G_{p}=\frac{k_{p}(1-0.5 L s)}{s(1+0.5 L s)}
$$

IMC controller for the above system consists of two parts. First part is the inverse of the stable portion of the process and second part is IMC filter. The numerator order of the IMC filter is equal to the number of unstable poles.

\section{Direct Synthesis Method}

In general, both the direct synthesis and IMC methods do not necessarily result in PI/PID controllers. However, by choosing the appropriate desired closed-loop response and using either a Pade' approximation or a power-series approximation for the time delay, PI/PID controllers can be derived for process models that are commonly used

in industrial applications.

The process transfer function is given by $\mathrm{Eq}$ (1). The controller transfer function is taken as

$$
\begin{aligned}
& G_{C}=k_{c}\left(1+\frac{1}{\tau_{I} s}+\tau_{D} s\right) \frac{1}{\left(1+\tau_{f} s\right)} \\
& \text { Where } \tau_{f}=\beta \tau_{D}
\end{aligned}
$$

\section{Stability Analysis Method}

Generally we will deal with a semi discrete model: discrete in space and continuous in time. In the time domain the model is given by ordinary differential equations (ODE) in time. Amplification Methods. Also called von Neumann stability analysis. Based on decomposition of motion into normal modes, often using Fourier analysis, and superposition. The analysis looks at the growth or decay of perturbations from one step to the next, and can be implemented using standard linear algebra procedures. It is local in nature, but so is the concept of stability. Amore severe restriction is that it strictly applies only to linear systems. Despite this limitation it is frequently applied to nonlinear systems through linearization. Energy Methods. Also known, notably in control theory, as Lyapunov methods. These look at the variation of certain function (or functional) measures of the motion amplitude. Often these are related to energy measures of various kinds, hence the name. Energy methods are not restricted to linear systems, but require the construction of suitable measures, and this has to be done case by case.

The process transfer function for pure integrator with time delay is given by equation Eq (1). The phase angle criterion for pure integrator with time delay is given by

$$
\phi(\omega)=-\frac{\pi}{2}-L \omega
$$


International Journal of Instrumentation and Control Systems (IJICS) Vol.7, No.3, July 2017

\section{Kharitonov's Theorem:}

The stability regions of the model parameters for the PID controller designed are calculated by Kharitonov's theorem considering uncertainty in one parameter at a time. Kharitonov's theorem is a result used in control theory to assess the stability of a dynamical system when the physical parameters of the system are not known precisely. When the coefficients of the characteristic polynomial are known, the Routh-Hurwitz stability criterion can be used to check if the system is stable (i.e. if all roots have negative real parts). Kharitonov's theorem can be used in the case where the coefficients are only known to be within specified ranges. It provides a test of stability for a so-called interval polynomial, while Routh-Hurwitz is concerned with an ordinary polynomial.

An interval polynomial is the family of all polynomials

$p(s)=a_{0}+a_{1} s^{1}+a_{2} s^{2}+\ldots+a_{n} s^{n}$ where each coefficient $a_{i} \in R$ can take any value in the specified intervals $\boldsymbol{t}_{i} \leq \boldsymbol{a}_{\boldsymbol{i}} \leq \boldsymbol{u}_{\boldsymbol{i}}$. It is also assumed that the leading coefficient cannot be zero: 0 \& $\left[\mathrm{r}^{152} \mathrm{si}^{13}\right]^{-}$.

An interval polynomial is stable (i.e. all members of the family are stable) if and only if the four so-called Kharitonov polynomials are stable.

$$
\begin{aligned}
& k_{1}(s)=l_{0}+l_{1} s^{1}+u_{2} s^{2}+u_{3} s^{3}+l_{4} s^{4}+l_{5} s^{5}+\cdots \\
& k_{2}(s)=u_{0}+u_{1} s^{1}+l_{2} s^{2}+l_{3} s^{3}+u_{4} s^{4}+u_{5} s^{5}+\cdots \\
& k_{3}(s)=l_{0}+u_{1} s^{1}+u_{2} s^{2}+l_{3} s^{3}+l_{4} s^{4}+u_{5} s^{5}+\cdots \\
& k_{4}(s)=u_{0}+l_{1} s^{1}+l_{2} s^{2}+u_{3} s^{3}+u_{4} s^{4}+l_{5} s^{5}+\cdots
\end{aligned}
$$

What is somewhat surprising about Kharitonov's result is that although in principle we are testing an infinite number of polynomials for stability, in fact we need to test only four. This we can do using Routh-Hurwitz or any other method. So it only takes four times more work to be informed about the stability of an interval polynomial than it takes to test one ordinary polynomial for stability.

Kharitonov's theorem is useful in the field of robust control, which seeks to design systems that will work well despite uncertainties in component behavior due to measurement errors, changes in operating conditions, equipment wear and so on.

\section{CONCLUSION}

In this paper we have derived optimal PID controller with time delay settings for a double integrating the process and compared the performance versus robustness trade-off with that obtained when varying the tuning parameter Tc for the SIMC-controller in (3). As seen from Figure 4, the SIMC controller has almost identical performance with the optimal, in particular for more robust designs (with lower value of Ms). This means that the simple SIMC PID tuning rules given in (3) are essentially the optimal. This is quite surprising, because the double integrating SIMC rules were originally derived in a fairly ad hoc manner, aiming more towards simplicity than optimality. Comparing the performance of the SIMC-rule with the optimal for a given robustness (Ms Value) shows that the SIMC-rule give settings close to the Pareto-optimal. This means that the room for improving the SIMC PI-rule is limited, at least for the first-order plus delay processes considered in this paper, and with a good trade-off between rejecting input and output (set-point) disturbances. In this article, also we discussed an IMC-based PID controller 
International Journal of Instrumentation and Control Systems (IJICS) Vol.7, No.3, July 2017

design method for several types of integrating process with time delay. Several important representative processes were considered in the simulation study, in order to demonstrate the superiority of the pro- posed method. The design method was based on the disturbance rejection and a setpoint filter was suggested to eliminate the over-shoo in the set-point response.

\section{ACKNOWLEDGEMENTS}

I would prefer to give thanks the researchers likewise publishers for creating their resources available. Additionally, I appreciative to commentator for their vital recommendations moreover I am conjointly grateful to reviewer for their valuable suggestions and also thank the college authorities for providing the required infrastructure and support.

\section{REFERENCES}

[1] Shamsuzzoha, Md. and Lee, M. 2008. PID controller design for integrating processes with time delay. Kor J Chem Eng, 25, 4: 637-645.

[2] Grimholt, C. and Skogestad, S. (2012). Optimal PI-control and verification of the SIMC tuning rule. In IFAC conference on Advances in PID controls (PID'12). The International Federation of Automatic Control.

[3] William L. Luyben.2009. Identification and Tuning of Integrating Processes with Dead time and Inverse Response.

[4] L. M. Waghmare G. M. Malwatkar. Design of PID Controllers for Improved Performance of Higher Order Systems.

[5] Di Ruscio, D. (2010). On Tuning PI Controllers for Integrating Plus Time Delay Systems. Modelling, Identification and Control 31(4), 145-164.

\section{AUTHOR}

Mr. B. S. Patil, Ph. D. Student, Instrumentation Department, AISSMS'S Polytechnic, Pune, Maharashtra 